\title{
Optimal Beaconing Control for Epidemic Routing in Delay-Tolerant Networks
}

\author{
Yong Li, Member, IEEE, Zhaocheng Wang, Senior Member, IEEE, Depeng Jin, Member, IEEE, Li Su, \\ Lieguang Zeng, and Sheng Chen, Fellow, IEEE
}

\begin{abstract}
Owing to the uncertainty of transmission opportunities between mobile nodes, the routing in delay-tolerant networks (DTNs) exploits the mechanism of opportunistic forwarding. Energy-efficient algorithms and policies for DTN are crucial to maximizing the message delivery probability while reducing the delivery cost. In this contribution, we investigate the problem of energy-efficient optimal beaconing control in a DTN. We model the message dissemination under variable beaconing rate with a continuous-time Markov model. Based on this model, we then formulate the optimization problem of the optimal beaconing control for epidemic routing and obtain the optimal threshold policy from the solution of this optimization problem. Furthermore, through extensive numerical results, we demonstrate that the proposed optimal threshold policy significantly outperforms the static policy with constant beaconing rate in terms of system energy consumption savings.
\end{abstract}

Index Terms-Beaconing control, delay-tolerant networks (DTNs), epidemic routing.

\section{INTRODUCTION}

$\mathbf{I}$ $\mathrm{N}$ delay-tolerant networks (DTNs), end-to-end paths from communication sources to destinations do not exist most of the time due to node mobility, wireless propagation effect, sparse node density, and other factors [1], [2]. Examples of DTNs include deep-space interplanetary networks [3], vehicular ad hoc networks [4], underwater networks [5], military networks [6], etc. DTNs are often found in critical application areas. For example, the vehicular DTN enables urgent data delivery between ambulances and the sensor nodes of highway infrastructure. In such networks, however, traditional ad hoc routing protocols, which rely on the end-to-end paths [7], may

Manuscript received September 23, 2010; revised May 14, 2011 and August 6, 2011; accepted October 23, 2011. Date of publication October 31, 2011; date of current version January 20,2012. This work was supported in part by the National Nature Science Foundation of China under Grant 61171065 and Grant 61021001, by the Chinese National Major Scientific and Technological Specialized Project under Grant 2010ZX03004-002-02, and by the Program for Changiiang Scholars and Innovative Research Team in University and Tsinghua National Laboratory for Information Science and Technology. The review of this paper was coordinated by Prof. H. Hassanein.

Y. Li, Z. Wang, D. Jin, L. Su, and L. Zeng are with the State Key Laboratory on Microwave and Digital Communications, Tsinghua National Laboratory for Information Science and Technology, Department of Electronic Engineering, Tsinghua University, Beijing 100084, China (e-mail: yong-li07@mails.tsinghua.edu.cn; zcwang@ tsinghua.edu.cn; jindp@ tsinghua. edu.cn; lisu@tsinghua.edu.cn; zenglg@tsinghua.edu.cn).

S. Chen is with the Department of Electronics and Computer Science, Faculty of Physical and Applied Sciences, University of Southampton, SO17 1BJ Southampton, U.K., and also with the Faculty of Engineering, King Abdulaziz University, Jeddah 21589, Saudi Arabia (e-mail: sqc@ecs.soton.ac.uk).

Color versions of one or more of the figures in this paper are available online at http://ieeexplore.ieee.org.

Digital Object Identifier 10.1109/TVT.2011.2174262 fail to work [1]. Therefore, a new routing mechanism called the store-carry-and-forward [8], [9] is proposed to provide communication. In this routing mechanism, when the next hop is not available for nodes to forward a message, the nodes will store the message in their local buffers, carry it along their movements, and forward it to other appropriate nodes when a transmission opportunity becomes available, which is referred to as a communication contact.

In many DTNs, energy is very limited. For example, in wireless mobile sensor networks for environmental sensing and wildlife behavior monitoring [10], sensor nodes are attached to animals, such as zebras [11] and deer [12]. These mobile sensor nodes form the DTN, and consequently, energy consumption will be a serious issue. Another example is a human network such as pocket-switched networking [13], where mobile users as the networking nodes use their mobile devices that are energy constrained. Therefore, it is necessary to design energy-efficient algorithms for message transmission. Furthermore, even for networks such as vehicular DTNs, where energy consumption is typically not a critical issue, unnecessary message transmissions and flooding are bad for the common welfare of the whole network since transmissions may also cause contentions to other users. Therefore, energy-efficient algorithms not only save the energy consumption but also influence the overall network throughput.

The system performance of a DTN depends on the overall energy budget. This energy budget should consider two main energy costs, which are beaconing energy for contact detection and communication energy for message transmission and reception [11], [14]. In fact, the nodes in a DTN are usually sparse, and they need to continuously discover the communication contacts. Consequently, nodes are beaconing during most of their lifetime and turn into message transmission state only when the contacts are detected. Therefore, the relay nodes spend substantial energy for periodical beaconing. How to schedule the energy consumption for beaconing is important to the whole system. The tradeoff between energy consumption and system performance in beaconing control is given as follows: If we increase the beaconing rate, we can obtain more communication contacts and, consequently, higher message transmission probability and smaller message delivery delay at the cost of higher energy consumption. By contrast, if the beaconing rate is too small, even when two nodes move within the transmission range, they may not detect the communication contact. Therefore, if the system overall energy budget is fixed, designing an energy-efficient beaconing control policy to balance this tradeoff is necessary. Previous 
works in the context of sensor networks have discussed the optimal activation times for sensor nodes [15], as well as the transmission control problem for the two-hop relaying scheme [14], which is a very simple forwarding mechanism in DTNs. To the best of our knowledge, no existing work has discussed the optimal beaconing control for the epidemic routing in the energy-constrained DTN.

In this contribution, we investigate the problem of optimal beaconing control for the DTN epidemic routing with the energy constraint for beaconing and message transmission. To achieve the maximum system performance in terms of message delivery probability under the condition that the energy is limited by a threshold, we control the beaconing rate of relay nodes. First, we introduce a continuous-time Markov framework to model the message dissemination of epidemic routing. Second, we formulate an optimization problem for the beaconing rate control. Based on the property of this optimization problem, we derive the optimal threshold policy, which maximizes the message delivery probability within the message lifetime while satisfying the given energy constraint. Finally, we demonstrate the efficiency of our optimal beaconing control policy by extensive results.

The organization of this paper is given as follows: In Section II, we model the system of message transmission. Based on this model, the problem of optimal beaconing control is formulated in Section III. In Section IV, we further analyze the formulated problem and investigate the optimal policy for the beaconing control. Performance evaluation is given in Section V, and we offer our concluding remarks in Section VI.

\section{SySTEM MODEL}

The DTN consists of a set of wireless mobile nodes denoted by $\mathbb{V}$, and the number of nodes in $\mathbb{V}$ is $N$, where $N>2$. Among these nodes, the source node generates messages to the network. We assume that a message is created in time 0 and that its lifetime is $T$. This means that, after time $T$, all the nodes in the network should discard it. Therefore, the goal of an optimal beaconing control policy is to transmit the message to the destination before time $T$. We investigate epidemic routing [16], which was one of the first routing algorithms proposed to deal with intermittent connectivity in mobile ad hoc networks [16]. In epidemic routing, when one node with the message comes into the communication range of another node that does not have the message, it forwards the message to this node. If this node is the destination, the communication ends. Otherwise, the epidemic forwarding continues. This way, this routing strategy floods the message over the whole network and attempts to send each message over all possible paths in the network. Therefore, it is an extreme-flooding-based routing strategy. Since it tries every possible path, epidemic routing delivers messages with minimum delivery delay and maximized delivery ratio. Consequently, epidemic routing is particularly useful for DTNs. This is the main reason that we chose epidemic routing to study the optimal beaconing control problem. Since the density of nodes is usual sparse in DTNs, nodes can communicate only when they have communication contacts. In our model, the contact process of any two nodes follows the Poisson process with the corresponding contact rate as its parameter, which is denoted by $\lambda$. Poisson-distributed contact rate and exponentially distributed intercontact time (ICT) are the key assumption, and based on this, we obtain the continuous Markov and fluid model latter. We now justify our chosen model.

We recognize that our model is only an approximation to the message transmission process in DTNs, because autocorrelated flows of contact events may exist in DTNs. Although some studies [17]-[19] have suggested the truncated power law for ICTs, these works actually reveal that the aggregated ICT follows a truncated power law, but the individual ICTs with the constraints can be better modeled by the exponential distributions with heterogeneous coefficients [20]-[24]. More specifically, [19] shows that the ICT has a truncated power law, with the exponential decay appearing in its tail after some cutoff point. However, generally, individual ICT follows the exponential distribution. For example, [20] reveals that, if ICTs are smaller than 1 week and each node pair has at least 20 contacts, then individual ICTs can be modeled by the exponential distributions with heterogeneous coefficients. Reference [21] shows that individual intermeeting time distribution can be shaped to be exponential by choosing an appropriate domain size with respect to a given time scale. Moreover, [22] models the ICTs of each individual human in mobile social networks by the Poisson process and experimentally validates this model by using the $\chi^{2}$ tests on real trace data. In [23] and [24], on the other hand, the ICTs between vehicles in vehicular ad hoc networks are modeled and reveal the exponential distribution of ICT between nodes by analyzing a large number of real car/taxi mobility traces. We further point out that the exponential model is widely used in many other recent works [25]-[29]. Based on the preceding discussion, we can justify that the adopted exponential distribution model is sufficiently accurate for studying the optimal beaconing control.

Since the network nodes are searching for other nodes that are in the communication range most of the time while the transmission time by contrast is very short, the energy spent in beaconing is substantial. Consequently, it is important to control the system beaconing energy. As previously stated, there exists a tradeoff between performance and energy consumption. If we use more power to beacon more often, the message will more quickly "infect" the network, but the energy consumption will be higher and nodes may empty their batteries sooner. On the other hand, by less frequently beaconing, the nodes' batteries will last longer, but message dissemination will be slower. Since the contact rate between nodes caused by physical mobility is $\lambda$, the maximal communication contact rate that can be detected by node beaconing is $\lambda$, which is achieved by consuming the maximal beaconing energy. If we reduce the beaconing energy by reducing the beaconing rate, we will get less communication contact. Therefore, we define $U(t)$ as the beaconing control for the energy consumption. Consequently, under policy $U(t)$, the message is transmitted upon the opportunistic contact with the rate of $U(t) \lambda$, and the dead rate of active nodes caused by empty battery is $U(t) \gamma$, where $\gamma$ is defined as the basic node death rate. Since, in reality, the node beaconing rate will be limited by the threshold, we assume that $u \leq U(t) \leq 1$, where $u$ is the minimum beaconing 
rate. We note that, when the nodes beacon at the maximal rate, the contact rate is $\lambda$. Therefore, here, we set the maximal beacon control to $U(t)=1$, which means that all the nodes beacon at the possible maximal energy consumption.

In this study, we consider the message transmission from the source to the destination. Let $Z(t)$ denote the number of message copies in all the nodes, including the original source node at time $t$. Obviously, $Z(0)=1$ and $Z=\{Z(t), t \geq 0\}$ is a stochastic process. From the definition of $Z(t)$, we note that $Z(t)$ satisfies the following equation:

$$
\begin{aligned}
P\left\{Z\left(t_{n+1}\right)=i_{n+1} \mid Z\left(t_{k}\right)\right. & \left.=i_{k}, 0 \leq k \leq n\right\} \\
& =P\left\{Z\left(t_{n+1}\right)=i_{n+1} \mid Z\left(t_{n}\right)=i_{n}\right\}
\end{aligned}
$$

where $0 \leq t_{0}<t_{1}<\cdots<t_{n+1}$ and $i_{k} \in\{1,2, \ldots, N\}$ for $0 \leq k \leq n+1$, and $P\{\bullet \mid \bullet\}$ denotes the conditional probability. Therefore, $Z$ is a continuous-time Markov chain. Markov chains can be approximated by ordinary differential equations (ODEs) [30], using a well-known technique known as the fluid model [25], which is widely adopted to model the epidemic forwarding [28], [31]. In the fluid model, the solution of the ODE converges in probability to the Markov chain's sample path. In this work, we use this model to characterize the message spreading.

We define $X(t)$ as the fraction of nodes that have a copy of the message at time $t$ and $Y(t)$ as the fraction of active nodes that have not received the copy by time $t$, respectively. Using the fluid model [25], we can obtain the ODEs for the system as follows:

$$
\begin{aligned}
& \frac{d X(t)}{d t}=\lambda X(t) Y(t) U(t) \\
& \frac{d Y(t)}{d t}=-(\lambda+\gamma) X(t) Y(t) U(t)
\end{aligned}
$$

where $\lambda$ is the contact rate, and $\gamma$ is the node death rate. The aforementioned term $\lambda X(t) Y(t) U(t)$ represents the increase in the fraction of nodes infected by the message since there are $X(t)$ fraction of nodes that can transmit the message to $Y(t)$ by the opportunistic contact of rate $\lambda$. The term $\gamma X(t) Y(t) U(t)$ represents the decrease in the number of active nodes caused by the death of nodes. From our system description, we have $X(0)=Z(0) / N=1 / N$ and $Y(0)=1$.

We now consider the energy consumption of the network. Since the wireless communication is the largest source of energy consumption in energy-limited devices [11], we consider only communication energy consumption and do not account for other energy consumption sources, such as computation and mobility. Existing studies show that the communication energy consumption mainly includes the transmitting and receiving consumption in the communication state, as well as the beaconing consumption in the idle state [11]. Based on this result, we calculate the amount of energy consumed by the network at time $T$. The first part of the energy consumption is proportional to the expected number of transmission times during the message's lifetime $T$, where the energy consumption of one transmission includes both the reception energy at the receiving node and the transmission energy at the transmitting node.
Therefore, it can be expressed as follows: $\alpha(N X(T)-1)$, where $\alpha$ is the system-specified positive constant that weighs the energy consumption of each transmission. The beaconing energy consumption is proportional to $U(t)$ and the fraction of active nodes. Thus, the total beaconing energy consumption up to time $T$ can be expressed as $\beta N \int_{0}^{T} U(t) Y(t) d t$, where $\beta$ is the system-specified positive constant that weight the energy consumption of each beaconing. By using (1), we have $\beta N \int_{0}^{T} U(t) Y(t) d t=(\beta N / \lambda) \log (X(T))$. Therefore, we can express the system total energy consumption at time $T$, which is denoted as $\phi(X(T))$, by the following expression:

$$
\phi(X(T))=\alpha N X(T)+\frac{\beta N}{\lambda} \log (X(T))-\alpha .
$$

Let $F(t)$ denote the probability that the message has been delivered to the destination at time $t$. Since our goal is to transmit the message to the destination before lifetime $T$, we should maximize $F(T)$, subject to the available system energy. Define the system total energy constraint as $\Psi$. Then, the problem of optimal beaconing rate control with the energy constraint for communication can be expressed as the following optimization problem:

$$
\begin{array}{ll}
\text { Maximize } & F(T) \\
\text { Subject to } & \phi(X(T)) \leq \Psi .
\end{array}
$$

Remark: Ideally, one would like to simultaneously maximize the probability that the message has been delivered to the destination at $T$, i.e., $F(T)$, and minimize the total energy consumed in doing so, i.e., $\phi(X(T))$. However, these are two conflicting objectives, and the optimization so formulated becomes a challenging multiobjective optimization problem. For such a multiobjective optimization, the optimization algorithm must generate a set of Pareto-optimal solutions, which should be ideally well distributed across the Pareto frontier [32]. Moreover, even when such a set of Paretooptimal solutions, which is represented in the objective space by $\left\{F^{\left(i_{*}\right)}(T), \phi^{\left(i_{*}\right)}\left(X^{\left(i_{*}\right)}(T)\right)\right\}$, can be obtained, a human decision or a preference structure has to be imposed as to choose which particular solution to use. This is usually achieved by first specifying what level of energy consumption, e.g., $\phi^{\left(i_{*}\right)}\left(X^{\left(i_{*}\right)}(T)\right)$, is to be allowed and then choosing the corresponding solution $F^{\left(i_{*}\right)}(T)$ from the Pareto-optimal set. In practice, therefore, the problem is often solved or approximated by setting the energy consumption limit or constraint $\phi(X(T)) \leq \Psi$ and maximizing $F(T)$, as is adopted in our approach of (4).

\section{Problem Formulation}

From the system model described by the ODEs, we divide (1) by (2) to yield

$$
\begin{aligned}
Y(t) & =-\frac{\lambda+\gamma}{\lambda}(X(t)-X(0))+Y(0) \\
& =-\frac{\lambda+\gamma}{\lambda} X(t)+C
\end{aligned}
$$


where $C=(\lambda+\gamma / \lambda) X(0)+Y(0)=1+(1 / N)+(\gamma / \lambda N)$. By substituting (5) into (1), we obtain the following ODE:

$$
\frac{d X(t)}{d t}=-((\lambda+\gamma) X(t)-C \lambda) X(t) U(t) .
$$

Solving this ODE yields

$$
X(t)=\frac{\lambda C}{\lambda+\gamma} \frac{D}{D+e^{-\lambda C \int_{0}^{t} U(t) d t}}
$$

where $D=(\lambda+\gamma) / \lambda C-(\lambda+\gamma)$. We have now obtained the fraction of nodes with the messages, i.e., $X(t)$. Based on $X(t)$, we can derive the expression for the message delivery probability as follows:

To derive $F(t)$, let us define $H(t)=1-F(t)$, which is the probability that the destination node has not received the message at time $t$. Then, we have

$$
H(t+\Delta t)=H(t) \cdot P\{\text { No delivery to dest. in } \Delta t \mid X(t)\} .
$$

Taking the expectation of the preceding expression yields $H(t+\Delta t)=H(t)\left(e^{-\lambda \Delta t}\right)^{N X(t)}$. From

$$
\lim _{\Delta t \rightarrow 0} \frac{H(t+\Delta t)-H(t)}{\Delta t}=\lim _{\Delta t \rightarrow 0} \frac{H(t)\left(\left(e^{-\lambda \Delta t}\right)^{N X(t)}-1\right)}{\Delta t}
$$

or $d H(t) / d t=-\lambda N H(t) X(t)$, we obtain $H(t)=H(0) e^{-\lambda N \int_{0}^{t} X(s) d s}$, where $H(0)=1$. Therefore, we have the following expression for $F(t)$ :

$$
F(t)=1-H(t)=1-e^{-\lambda N \int_{0}^{t} X(s) d s} .
$$

Thus, the optimization problem (4) for the optimal beaconing control is specified by

$$
\begin{array}{ll}
\text { Maximize } & 1-e^{-\lambda N \int_{0}^{T} X(s) d s} \\
\text { Subject to } & \left\{\begin{array}{l}
X(T)=\frac{\lambda C}{\lambda+\gamma} \frac{e^{\lambda C} \int_{0}^{T} U(t) d t}{e^{\lambda C} \int_{0}^{T} U(t) d t}+\frac{1}{D} \\
\alpha N X(T)+\frac{\beta N}{\lambda} \log (X(T))-\alpha \leq \Psi \\
u \leq U(t) \leq 1 .
\end{array}\right.
\end{array}
$$

\section{Optimal Beaconing Solution}

In this section, we derive the optimal solution for the formulated optimization problem of (9).

Lemma 1: Both $\phi(X(T))$ and $F(T)$ are monotonously increasing functions of $X(t)$.

Proof: Denote $\quad x=X(T)$. From $\quad \phi(x)=\alpha N x+$ $(\beta N / \lambda) \log (x)-\alpha$, we have

$$
\frac{d \phi(x)}{d x}=\alpha N+\frac{\beta N}{\lambda x}>0 .
$$

Therefore, $\phi(X(T))$ is a monotonously increasing function of $x$. Similarly, since $F(T)=F(X(T))=F(x)=1-e^{-\lambda N T x}$, we have

$$
\frac{d F(x)}{d x}=\lambda N T e^{-\lambda N T x}>0 .
$$

Therefore, $F(T)$ is a monotonously increasing function of $x$. This proves the lemma.

Based on Lemma 1, there exists one and only one real value $\psi$ such that $X(T) \leq \psi$. Therefore, the system energy constraint can be expressed as

$$
\frac{D}{D+e^{-\lambda C \int_{0}^{T} U(t) d t}} \leq \frac{\psi(\lambda+\gamma)}{\lambda C} .
$$

Furthermore, the monotonously increasing nature of $\phi(X(T))$ and $F(T)$ allows us to transform the optimization problem (9) into the following equivalent but simpler form:

$$
\begin{aligned}
& \text { Maximize } \int_{0}^{T} X(t) d t \\
& \text { Subject to }\left\{\begin{array}{l}
\int_{0}^{T} U(t) d t \leq \frac{1}{\lambda C} \log \frac{\psi(\lambda+\gamma)}{(\lambda C-\psi(\lambda+\gamma)) D} . \\
u \leq U(t) \leq 1
\end{array}\right.
\end{aligned}
$$

Lemma 2: Given a policy $U(t)$, consider policy $U^{\prime}(t)$ that satisfies $U^{\prime}(t) \succeq U(t)$, which means that $U^{\prime}(\tau) \geq U(\tau)$ for any $\tau \in[0, T]$. Then, $F^{\prime}(T) \geq F(T)$ holds.

Proof: Because $U^{\prime}(t) \succeq U(t)$, for a given $T, \int_{0}^{T} U^{\prime}(t) d t \geq$ $\int_{0}^{T} U(t) d t$. As $D / D+e^{-\lambda C x}$ increases with $x$, it holds that $X^{\prime}(T) \geq X(T)$. Since $F(T)$ is an increasing function of $X(T)$, it immediately follows that $F^{\prime}(T) \geq F(T)$.

We have the following two theorems for the optimization problem (11):

Theorem 1: The optimal policy for beaconing control, which is denoted as $U^{*}(t)$, saturates the constraint, i.e., $\int_{0}^{T} U^{*}(t) d t=$ $W$, where

$$
W=\max _{u \leq U(t) \leq 1} \int_{0}^{T} U(t) d t=\frac{1}{\lambda C} \log \frac{\psi(\lambda+\gamma)}{(\lambda C-\psi(\lambda+\gamma)) D}
$$

is the maximum possible area under $U(t)$ over interval $[0, T]$, which is the direct consequence of (10).

Proof: Consider policy $U(t)$, which is different from the optimal policy $U^{*}(t)$ and does not saturate the energy constraint, i.e., $\int_{0}^{T} U(t) d t<W$. Then, $\exists \varepsilon>0$, such that $\int_{0}^{T} U(t) d t+\varepsilon \leq W$. Consequently, we can obtain a new policy denoted by $U^{\prime}(t)=U(t)+\varepsilon / T$, which satisfies $\int_{0}^{T} U^{\prime}(t) d t \leq W$. Obviously, $U^{\prime}(t) \succeq U(t)$. According to Lemma 2, we have $F^{\prime}(T) \geq F(T)$. Thus, $U^{\prime}(t)$, which satisfies the energy constraint, is a better policy than $U(t)$. This proves the theorem that the optimal policy saturates the constraint.

Theorem 2: The optimal beaconing control policy $U^{*}(t)$ for the optimization problem (11) is

$$
\begin{array}{cl}
\text { If } T<W: & U^{*}(t)=1 \text { for } 0 \leq t \leq T \\
\text { If } W \leq T \leq \frac{W}{u}: & U^{*}(t)= \begin{cases}1, & t \leq \frac{W-T u}{1-u} \\
u, & t>\frac{W-T u}{1-u}\end{cases} \\
\text { If } T>\frac{W}{u}: & \text { there exists no solution. }
\end{array}
$$


Proof: The solution (13) and the nonsolution (15) are obvious. Consider the case $W \leq T \leq(W / u)$. Denote $P(t)=$ $\int_{0}^{t} U(\tau) d \tau$, which is a monotonously increasing function of $U(t)$ for $0 \leq t \leq T$. Note that the optimization objective in (11) is $\int_{0}^{T} X(t) d t$. From the expression of $X(t)$ in (7), it is obvious that $\int_{0}^{T} X(t) d t$ increases with $\{P(t), 0 \leq t \leq T\}$. Therefore, to maximize the objective $\int_{0}^{T} X(t) d t,\{P(t), 0 \leq t \leq T\}$ and, consequently, $\{U(t), 0 \leq t \leq T\}$ should be made as large as possible. Thus, the optimal policy $u \leq U^{*}(t) \leq 1$ should take the largest value 1 as long as possible while ensuring that $P^{*}(T)$ saturates the constraint, i.e., $P^{*}(T)=W$. Without loss of generality, assume that $U^{*}(t)=1$ for $0 \leq t \leq t_{\max }$ and $U^{*}(t)=u$ for $t_{\max }<t \leq T$. Solving $\int_{0}^{t_{\max }} 1 d t+\int_{t_{\max }}^{T} u d t=W$ yields $t_{\max }=W-T u / 1-u$. This proves the solution (14).

\section{Performance Evaluation}

Before we evaluated the proposed optimal beaconing control policy, we had first validated the model used in deriving this optimal beaconing control policy.

\section{A. Model Validation}

We evaluated the accuracy of our model by comparing the theoretical results obtained based on model (7) with the simulation results, which were obtained by simulating the message dissemination under the epidemic routing with different mobility models. Without loss of generality, we set the forwarding policy $U(t)=1$ and the node death rate $\gamma=0$, because our goal was to verify the accuracy of the theoretical model.

We first simulated the network with different network sizes of $N$ from 100 to 1000 , where the Poisson process with parameter $\lambda=3.71 \times 10^{-6} \mathrm{~s}^{-1}$ was used to generate node contact events. This value of $\lambda$ was obtained from the vehicular ad hoc network model discussed in [24]. The same $\lambda$ value was used in the theoretical model (7). At the beginning of the simulation, $20 \%$ of the nodes was chosen to be infected. The theoretical and simulation results obtained for the infected node ratio as a function of time $t$ are plotted in Fig. 1, where the label "Theoretical" refers to $X(t) / N$, with $X(t)$ calculated according to (7), whereas the label "Simulation" refers to the infected node ratio generated by the network simulation. As expected, regardless of the size of the simulated network, the number of message-infected nodes computed from our model (7) agreed with that obtained by the network simulation; for instance, in this case, both our model and the simulated network assumed the Poisson-distributed contact process.

We then used the synthetic mobility model Random WayPoint (RWP) and the real-world map-driven DTN model (MAP) with pedestrians and transportation systems in the opportunistic network environment (ONE) simulator [33]. Note that the RWP is a mobility model, whereas the MAP in the ONE simulator represents the realistic DTN environment, where the contact rate is not governed by the Poisson process. We set $N=200$, chose initially $20 \%$ of the nodes to be infected by one message, and used the default mobility model settings in the ONE simulator. When choosing the initial infected nodes, we gave a chosen

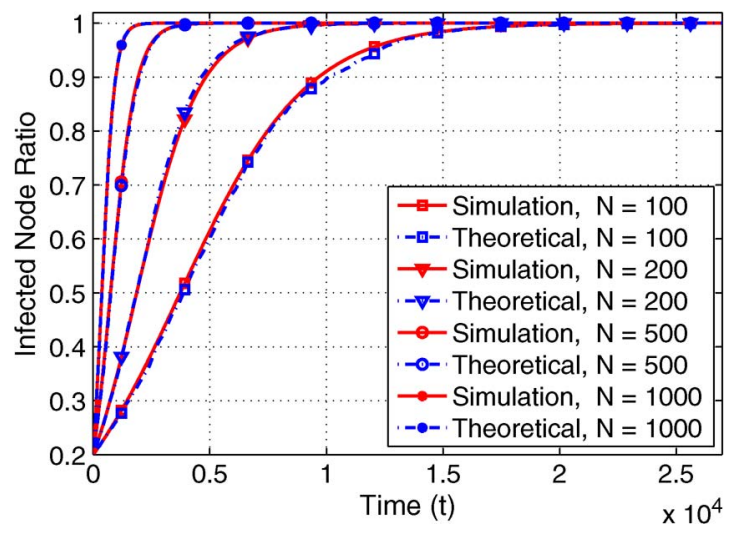

Fig. 1. Theoretical and simulation result comparison in terms of the infected node ratio as a function of time $t$ for the network assuming the Poissondistributed contact process and with different network sizes.

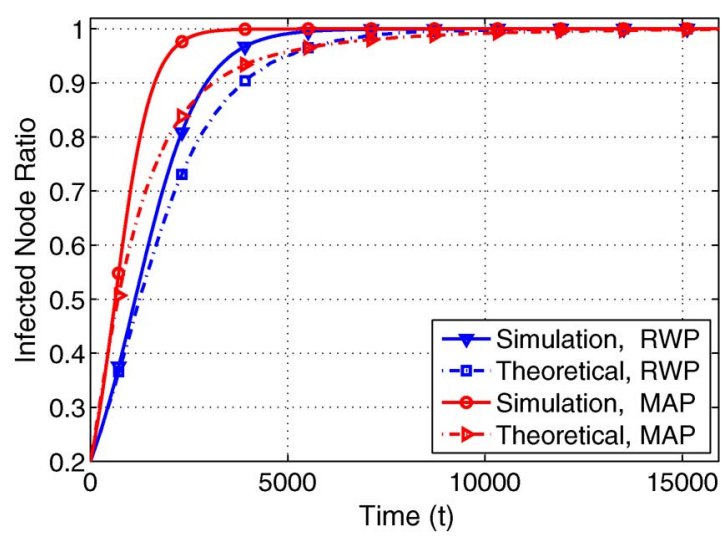

Fig. 2. Theoretical and simulation result comparison in terms of the infected node ratio as a function of time $t$ for the two different mobility networks with the fixed network size of $N=200$.

weight of 1-8 to each node by dividing the nodes into eight groups according to their distances to the source node obtained from their initial locations. For each of the two DTN simulation environments, we fitted an exponential distribution to the ICT generated and used the obtained $\lambda$ value in our model (7). Fig. 2 plots the message-infected node ratios as a function of time $t$ obtained by simulation and theoretical calculation (7) for the two mobility networks, respectively. It can be seen from Fig. 2 that the theoretical calculations did approximate the network simulation results reasonably well. To quantitatively analyze the results, we calculated the average deviation between the theoretical and simulation results, which is defined as

$$
\text { Average Deviation }=\frac{1}{T} \int_{0}^{T} \frac{\left|X^{(\mathrm{The})}(t)-X^{(\operatorname{Sim})}(t)\right|}{X^{(\operatorname{Sim})}(t)} d t
$$

where $X^{(\text {The) }}(t)$ denotes the number of message-infected nodes calculated according to (7), whereas $X^{(\operatorname{Sim})}(t)$ is the number of message-infected nodes generated by the network simulation. The average deviations between the theoretical calculations and the simulation results were about $5.7 \%$ and $8.6 \%$, respectively, for the RWP and MAP DTN simulation environments. 

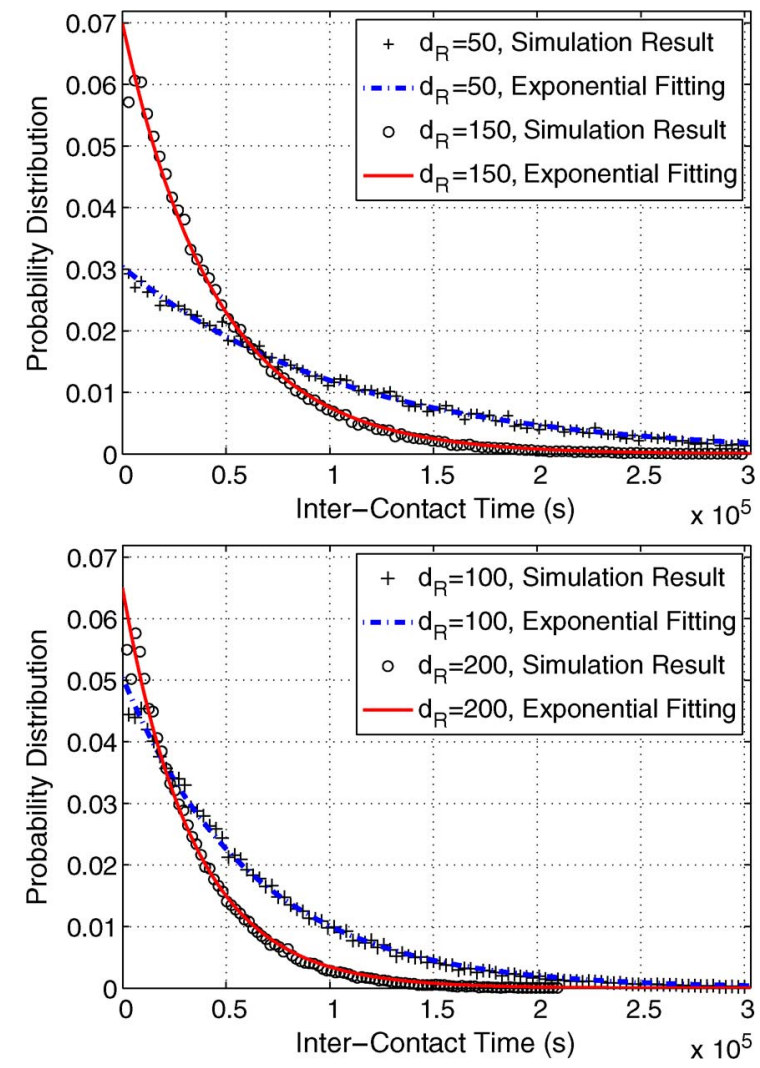

Fig. 3. Fitting the exponential distribution to the data of ICT obtained by the RWP simulation with different transmission ranges $d_{R}$.

The aforementioned results clearly demonstrated that the contact rates of realistic mobility models do not strictly follow the Poisson distribution, and our assumption of the exponentially distributed ICT is only an approximation. Therefore, we further investigated the accuracy of our model. To know the influence of the transmission range, which is denoted as $d_{R}$, on the ICT distribution, we set $d_{R}$ to $50,100,150$, and $200 \mathrm{~m}$, respectively. Under the same RWP and MAP simulation environments with these different transmission ranges, we obtained the actual simulation data of ICT distribution using the ONE simulator and then fitted the exponential distribution to the simulation data. The results are shown in Figs. 3 and 4 for the RWP and MAP simulations, respectively. Visually, we observe that the exponential distribution did fit the simulation data well, and the transmission range $d_{R}$ generally did not have a major influence on the fitting quality. To quantitatively provide the fitting accuracy, we employed the adjusted R-square statistics [34] as the goodness of fit, which is defined as the percentage of the variation between the simulation data and the fitting results. Table I lists the adjusted R-square statistics of the exponential fitting to the simulation data of ICT obtained, where the adjusted R-square statistics were computed with Matlab Curve Fitting Toolbox. It can be seen from Table I that the average adjusted R-square statistics was above $99 \%$ for the RWP, and it was about $94 \%$ for the MAP. This demonstrates the accuracy of the Poisson contact rate assumption adopted in our model.

In the aforementioned model validation, which was presented in Figs. 1 and 2, one message transmission scenario was used since model (7) represents the limit case of one message
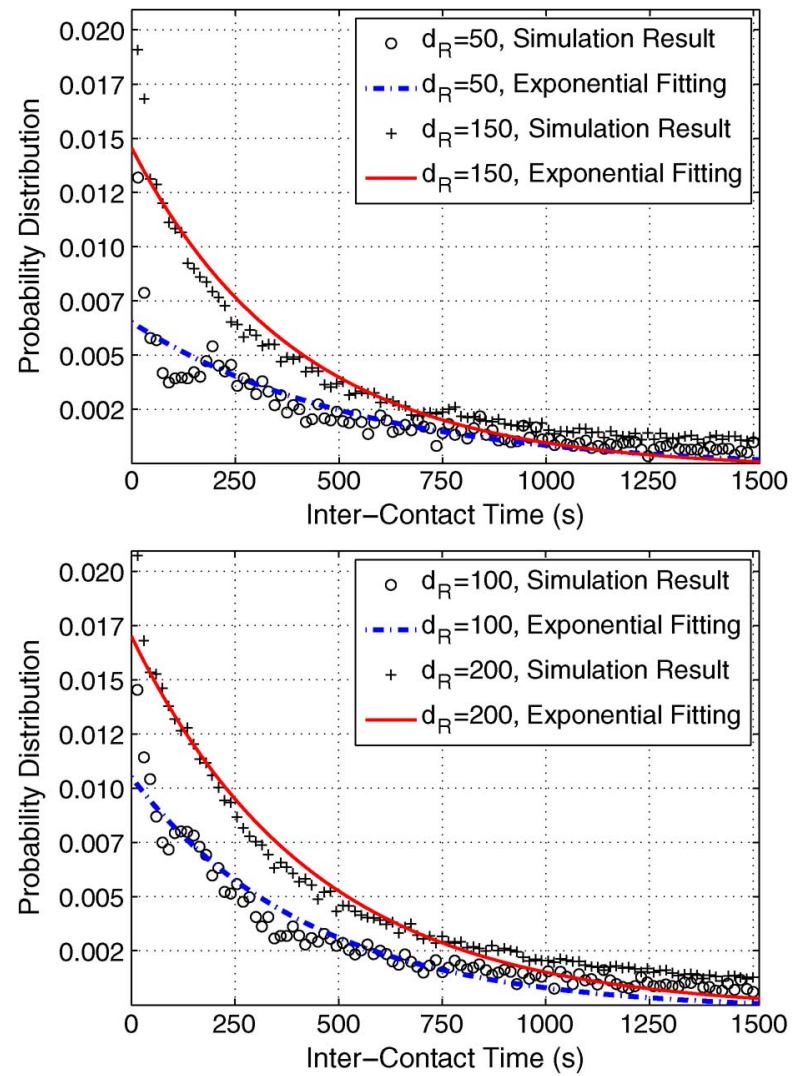

Fig. 4. Fitting the exponential distribution to the data of ICT obtained by the MAP simulation with different transmission ranges $d_{R}$.

TABLE I

Adjusted R-SQUare STATISTICS OF THE ExponEnTIAL FitTing TO THE DATA of InTERCONTACT Time ObTAINED From the Two DifFERENT MOBILITY NETWORK SiMULATION ENVIRONMENTS WITH DIFFERENT TRANSMISSION RANGES

\begin{tabular}{|c|cccc|}
\hline Network Model & $d_{R}=50$ & $d_{R}=100$ & $d_{R}=150$ & $d_{R}=200$ \\
\hline RWP & $99.44 \%$ & $99.73 \%$ & $99.56 \%$ & $99.31 \%$ \\
\hline MAP & $91.33 \%$ & $93.05 \%$ & $96.11 \%$ & $96.57 \%$ \\
\hline
\end{tabular}

transmission. According to the definition (8), we can obtain the expected message transmission delay for the transmission of a large number of messages, which is denoted by $D_{\mathrm{a}}$, as follows:

$$
D_{\mathrm{a}}=\int_{0}^{\infty}(1-F(t)) d t
$$

given a constant beaconing policy. Using the expected message transmission delay, we were able to further validate our model in a realist multiple-message transmission scenario. We transmit 10000 messages from the source to the destination under the same RWP and MAP simulation environments previously introduced. It is worth emphasizing again that the RWP and MAP represent the synthetic mobility model and the realistic DTN environments, respectively, which do not impose the Poisson distributed contact rate. We varied the constant beaconing policy $U(t)$ from 0.05 to 1.0 and recorded the average message transmission delays obtained in the corresponding network simulations. The simulation results are then compared to the theoretical calculations obtained using (17) in Fig. 5, where it can be seen that the message transmission delay 


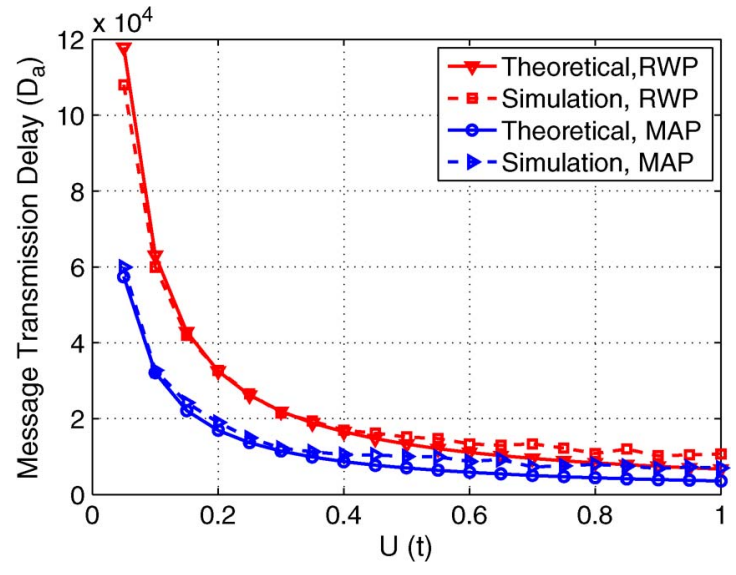

Fig. 5. Theoretical and simulation result comparison in terms of the expected message transmission delay as a function of $U(t)$ for the two different mobility networks.

computed from our model agrees well with the simulation result. Using a similar definition to (16), the average deviation between the simulated message transmission delay results and the theoretical calculations is about $5.1 \%$. Interestingly, this deviation obtained under the more realistic multiple-message transmission scenario appears to be smaller than that obtained previously under the one-message transmission scenario when investigating the infected node ratio. This further indicates the accuracy of our model.

In the aforementioned model validation, we have extensively demonstrated that our assumption of Poisson contact rate and the resulting message transmission model are sufficiently accurate to approximate or model the message dissemination of epidemic routing in DTNs. Thus, we can confidently use the continuous-time Markov and fluid model to study the optimal beaconing control problem.

\section{B. Evaluation Settings and Results}

We used the continuous-time Markov and fluid model to represent the DTN application to the vehicular ad hoc network that was based on the real motion traces from about 2100 operational taxis for about one month in Shanghai city collected by Global Positioning System [35], in which the location information of the taxis were recorded every $40 \mathrm{~s}$ within the area of $102 \mathrm{~km}^{2}$. By analyzing large amounts of the trace data given in [35], [23] and [24] found that the distribution of the ICT between taxis followed the exponential distribution on a large time scale. Moreover, Zhu et al. [24] performed a least-squares fitting to identify the exponential parameter and found that the ICT was well approximated by the exponential distribution of $P\{X>t\}=e^{-3.71 \times 10^{-6} t}$. Therefore, in our model, we set parameter $\lambda=3.71 \times 10^{-6} \mathrm{~s}^{-1}$. Since, in typical practical DTNs, the number of nodes is in the hundreds, we set $N$ to 200 in our model. To evaluate the system performance enhancement achievable by the proposed optimal beaconing policy in the systems with low-energy resources, we set energy constraint $\psi$ to a low value of 40 , which implies that only $20 \%$ of the nodes are allowed to be involved in the message transmission. Thus, unless otherwise specifically stated, the default values for the

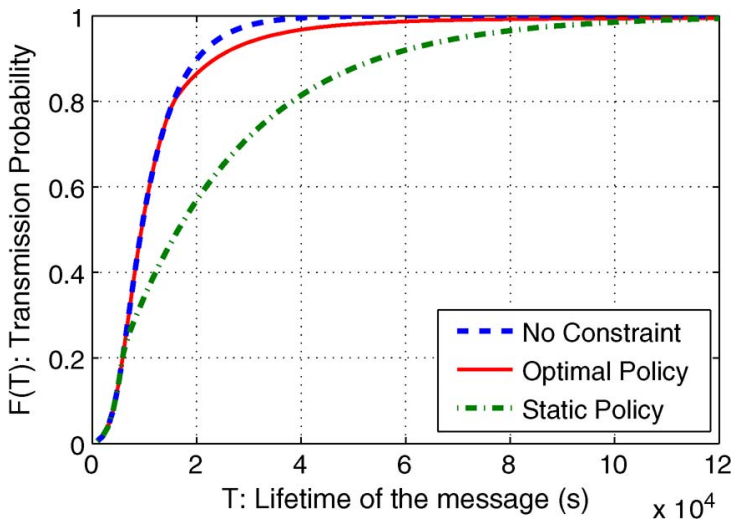

Fig. 6. Comparison of the delivery probabilities as a function of the message lifetime for three beaconing policies.

parameters in the numerical simulation of our model were set to $u=0.1, \gamma=1.5 \times 10^{-5}, N=200$, and $\psi=40$.

Since our work is the first to study the problem of the optimal beaconing control in epidemic routing, there exist no other optimal beaconing policies proposed in literature that can be used for comparison purposes. For the comparison with our proposed optimal beaconing control policy, which was denoted as Optimal Policy, we considered a static policy that used a constant beaconing rate. According to Theorem 1, the optimal static policy is defined by $U(t)=W / T$, which we referred to as Static Policy. Furthermore, we also considered a benchmark policy that does not have energy constraint and beacons at the maximum rate, i.e., $U(t)=1$ and $\psi=\infty$, which was simply referred to as No Constraint. Obviously, this No Constraint policy offers the maximum performance upper bound, but it is unrealistic.

Fig. 6 compares the transmission probabilities $F(T)$ as the function of massage lifetime $T$ achieved by the three beaconing policies. As expected, the message transmission probability $F(T)$ increased as the message lifetime $T$ increased. However, given the energy constraint $\psi=40$, the Static Policy could only attain an $F(T)$ value of about $80 \%$ when $T=4 \times 10^{4} \mathrm{~s}$, and its $F(T)$ value was still less than 1 , even when $T=10^{5} \mathrm{~s}$. By contrast, our proposed Optimal Policy with energy constraint $\psi=40$, which only allowed $40 / 200=20 \%$ of the nodes to be "infected" by the message, attained a much higher $F(T)$ value, which was observed to be very close to that obtained by the No Constraint policy with $U(t)=1$ and $\psi=\infty$. This clearly demonstrated the efficiency of our proposed optimal beaconing policy.

The results of the delivery probability at $T=2 \times 10^{4} \mathrm{~s}$ as a function of node death rate $\gamma$ are plotted in Fig. 7. Obviously, the No Constraint policy of $U(t)=1$ and $\psi=\infty$ could achieve the best delivery probability, but this policy is impractical to implement. As expected, the message transmission probability of the No Constraint policy decreased as the nodes death rate increased, because a larger $\gamma$ implied that less nodes were in the active state to transmit the message in this case. By contrast, $F(T)$ of the proposed Optimal Policy and Static Policy increased with $\gamma$. The reason was that, with these two policies, the energy constraint was taken into consideration; therefore, the system energy was well scheduled. Specifically, for the 


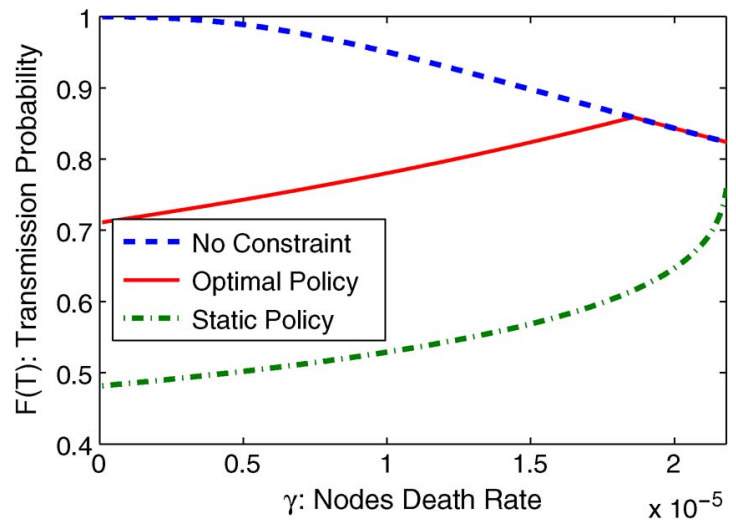

Fig. 7. Comparison of the delivery probabilities at $T=2 \times 10^{4} \mathrm{~s}$ as a function of the node death rate for three beaconing policies.

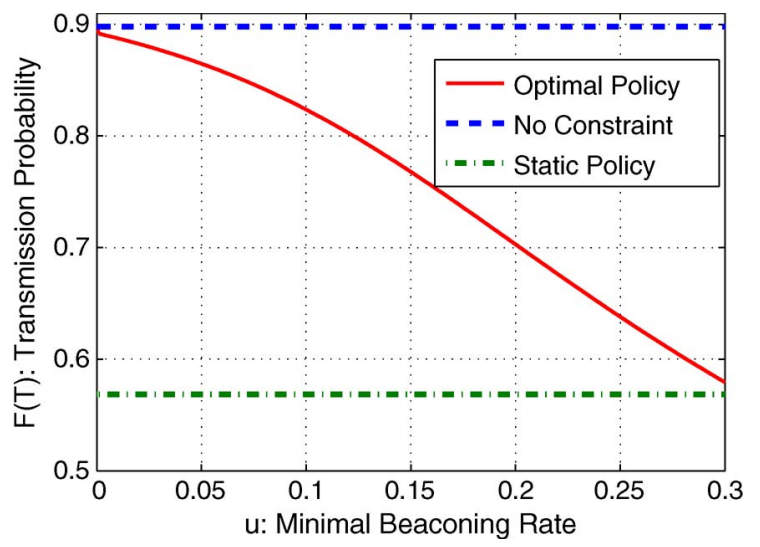

Fig. 8. Comparison of the delivery probabilities at $T=2 \times 10^{4} \mathrm{~s}$ as a function of the minimum beaconing rate for three beaconing policies.

Optimal Policy, the system energy was scheduled by optimal threshold policy $U(t)$ expressed in (13)-(15) for the given $\gamma$, whereas in the Static Policy, $U(t)$ was set to $W / T$, where $W$ can be shown to be a monotonously increasing function of $\gamma$. Compared with the static policy, our optimal threshold policy attained about $20 \%$ higher transmission probability.

By changing the value of the minimum beaconing rate $u$, we obtained the results shown in Fig. 8. Obviously, the minimum beaconing rate had no effect on the performance of the Static Policy and No Constraint policy. The transmission probability achieved by our Optimal Policy decreased as $u$ increased. This was because, when $u$ was large, the time for keeping the beaconing rate $U(t)=1$ was short; therefore, the message was transmitted with long delay and low delivery probability. From Fig. 8, it could be seen that the proposed Optimal Policy achieved a considerably higher transmission probability than the Static Policy, and this further demonstrated the effectiveness of the proposed Optimal Policy.

Fig. 9 compares the minimum energy constraint $\psi$ required by the Optimal Policy to achieve the given delivery probability $F(T)$ at $T=2 \times 10^{4} \mathrm{~s}$ with that required by the Static Policy. Clearly, the proposed Optimal Policy was much more energy efficient than the Static Policy. To show the energy saving of the Optimal Policy over the No Constraint policy, we considered the number of message transmissions or nodes infected according to the achieved delivery probability $F(t)$ with the

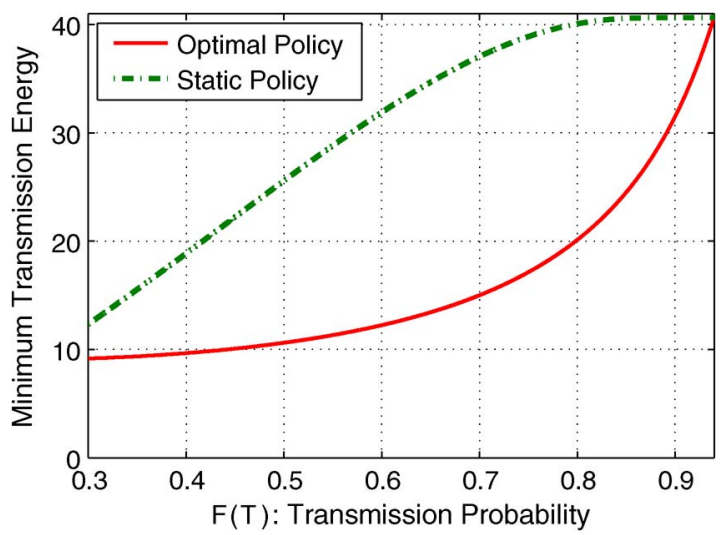

Fig. 9. Comparison of the minimum energy constraint values $\psi$ required to achieve the given delivery probability $F(T)$ at $T=2 \times 10^{4} \mathrm{~s}$ for two beaconing policies.

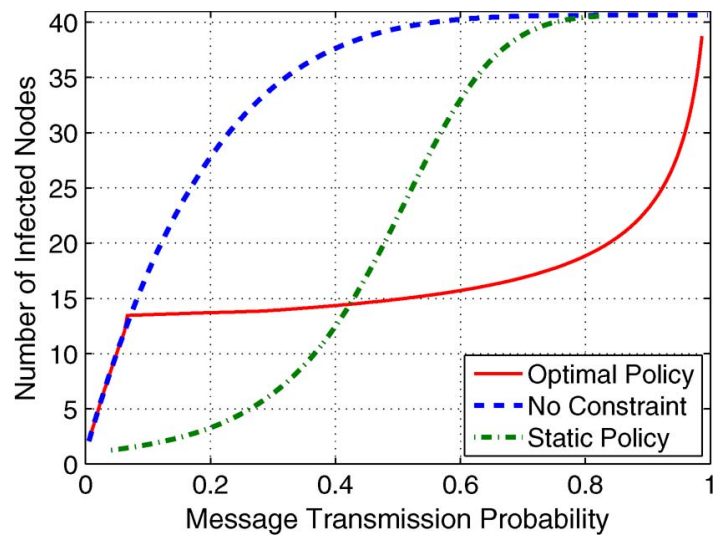

Fig. 10. Comparison of the numbers of message transmissions or infected nodes according to the achieved delivery probability $F(t)$ with the increase of time $t$ from $0 \mathrm{~s}$ to $1 \times 10^{5} \mathrm{~s}$ for three beaconing policies.

increase of time $t$ from $0 \mathrm{~s}$ to $1 \times 10^{5} \mathrm{~s}$. The results obtained by the three beaconing policies are shown in Fig. 10. As expected, the numbers of message transmissions of both the Static Policy and Optimal Policy to attain the same $F(t)$ were smaller than that of the No Constraint policy, as the No Constraint policy was most energy inefficient. For $F(t) \leq 0.43$, the Static Policy required a smaller number of message transmissions, compared with the Optimal Policy. However, for $F(t)>0.43$, the number of message transmissions of the Static Policy quickly increased, and our Optimal Policy required a much smaller number of message transmissions to attain the same message transmission probability.

\section{CONCLUSION}

We have introduced a continuous-time Markov and fluid model to analyze the problem of optimal beaconing rate control in the DTN. We have solved this optimization problem and derived the optimal threshold beaconing policy. We have performed an extensive model validation to support our assumption that the Poisson contact rate and the resulting message transmission model are sufficiently accurate to model the message dissemination of epidemic routing in DTNs. Our numerical results have demonstrated the efficiency of the 
proposed optimal beaconing policy in enhancing the system performance, in terms of message delivery probability and system energy consumption.

In the derivation of our optimal beaconing policy, we have explicitly assumed epidemic routing in DTNs. Therefore, our optimal threshold policy may no longer be optimal or may not even be applicable for the DTNs that use other routing schemes. Our future work is to study the optimal beaconing policy that is independent of the routing algorithm. Although the continuous Markov and fluid model is capable of modeling the data transmission in epidemic routing, it is still an approximation. Therefore, it is highly desirable to investigate a combined discrete-event and continuous model to study the non-Poisson-distributed contact rate in the future.

\section{REFERENCES}

[1] K. Fall, "A delay-tolerant network architecture for challenged Internets," in Proc. Conf. Appl., Technol., Architectures, Protocols Comput. Commun., New York, Aug. 25-29, 2003, pp. 27-34.

[2] K. Fall and S. Farrell, "DTN: An architectural retrospective," IEEE J. Sel. Areas Commun., vol. 26, no. 5, pp. 828-836, Jun. 2008.

[3] S. Burleigh, A. Hooke, L. Torgerson, K. Fall, V. Cerf, B. Durst, K. Scott, and $\mathrm{H}$. Weiss, "Delay-tolerant networking: An approach to interplanetary Internet," IEEE Commun. Mag., vol. 41, no. 6, pp. 128-136, Jun. 2003.

[4] J. Burgess, B. Gallagher, D. Jensen, and B. N. Levine, "Maxprop: Routing for vehicle-based disruption-tolerant networks," in Proc. 25th IEEE INFOCOM, Barcelona, Spain, Apr. 23-29, 2006, pp. 1-11.

[5] N. Chirdchoo, W. S. Soh, and K. C. Chua, "Aloha-based MAC protocols with collision avoidance for underwater acoustic networks," in Proc. 26th IEEE INFOCOM, Anchorage, AK, May 6-12, 2007, pp. 2271-2275.

[6] R. Krishnan, P. Basu, J. Mikkelson, C. Small, R. Ramanathan, D. W. Brown, J. R. Burgess, A. L. Caro, M. Condell, N. C. Goffee, R. R. Hain, R. E. Hansen, C. E. Jones, V. Kawadia, D. P. Mankins, B. I. Schwartz, W. T. Strayer, J. W. Ward, D. P. Wiggins, and S. H. Polit, "The spindle disruption-tolerant networking system," in Proc. IEEE MILCOM, Orlando, FL, Oct. 29-31, 2007, pp. 1-7.

[7] M. S. Blumenthal and D. D. Clark, "Rethinking the design of the Internet: The end-to-end arguments vs. the brave new world," ACM Trans. Internet Technol., vol. 1, no. 1, pp. 70-109, Aug. 2001.

[8] S. Jain, K. Fall, and R. Patra, "Routing in a delay tolerant network," in Proc. Conf. Appl., Technol., Architectures, Protocols Comput. Commun., Portland, OR, Aug. 30-Sep., 3, 2004, pp. 145-158.

[9] Z. Zhang, "Routing in intermittently connected mobile ad hoc networks and delay tolerant networks: Overview and challenges," IEEE Commun. Surveys Tuts., vol. 8, no. 1, pp. 24-37, First Quarter, 2006.

[10] J. Eriksson, L. Girod, B. Hull, R. Newton, S. Madden, and H. Balakrishnan, "The pothole patrol: Using a mobile sensor network for road surface monitoring," in Proc. 6th Int. Conf. Mobile Syst., Appl. Serv., Breckenridge, CO, Jun. 17-20, 2008, pp. 29-39.

[11] M. Martonosi, "Embedded systems in the wild: ZebraNet software, hardware, and deployment experiences," ACM SIGPLAN Notices, vol. 41, no. 7 , p. 1, Jul. 2006.

[12] DeerNet. Project, 2009. [Online]. Available: http://www.nsf.gov/news/ specialreports/animals/index.jsp

[13] P. Hui, J. Crowcroft, and E. Yoneki, "BUBBLE rap: Social-based forwarding in delay tolerant networks," in Proc. 9th ACM MobiHoc, Hong Kong, May 26-30, 2008, pp. 241-250.

[14] E. Altman, A. P. Azad, T. Basar, and F. De Pellegrini, "Optimal activation and transmission control in delay tolerant networks," in Proc. 29th IEEE INFOCOM, San Diego, CA, Mar. 14-19, 2010, pp. 106-110.

[15] K. Kar, A. Krishnamurthy, and N. Jaggi, "Dynamic node activation in networks of rechargeable sensors," IEEE/ACM Trans. Netw., vol. 14, no. 1, pp. 15-26, Feb. 2006.

[16] A. Vahdat and D. Becker, "Epidemic routing for partially-connected ad hoc networks," Dept. Comput. Sci., Duke Univ., Durham, NC, Tech. Rep. CS-200006, 2000

[17] P. Hui, A. Chaintreau, J. Scott, R. Gass, J. Crowcroft, and C. Diot, "Pocket switched networks and human mobility in conference environments," in Proc. WDTN, Philadelphia, PA, Aug. 22-26, 2005, pp. 244-251.
[18] A. Chaintreau, P. Hui, J. Crowcroft, C. Diot, R. Gass, and J. Scott, "Impact of human mobility on the design of opportunistic forwarding algorithms," IEEE Trans. Mobile Comput., vol. 6, no. 6, pp. 606-620, Jun. 2006.

[19] T. Karagiannis, J. Y. Le Boudec, and M. Vojnovi, "Power law and exponential decay of inter contact times between mobile devices," IEEE Trans. Mobile Comput., vol. 9, no. 10, pp. 1377-1390, Oct. 2010.

[20] V. Conan, J. Leguay, and T. Friedman, The Heterogeneity of Inter-Contact Time Distributions: Its Importance for Routing in Delay Tolerant Networks, Sep. 2006, arXiv:cs/0609068.

[21] H. Cai and D. Y. Eun, "Crossing over the bounded domain: From exponential to power-law intermeeting time in mobile ad hoc networks," IEEE/ACM Trans. Netw., vol. 17, no. 5, pp. 1578-1591, Oct. 2009.

[22] W. Gao, Q. Li, B. Zhao, and G. Cao, "Multicasting in delay tolerant networks: A social network perspective," in Proc. 10th ACM MobiHoc, New Orleans, LA, May 18-21, 2009, pp. 299-308.

[23] K. Lee, Y. Yi, J. Jeong, H. Won, I. Rhee, and S. Chong, "Maxcontribution: On optimal resource allocation in delay tolerant networks," in Proc. 29th IEEE INFOCOM, San Diego, CA, Mar. 14-19, 2010, pp. 1-9.

[24] H. Zhu, L. Fu, G. Xue, Y. Zhu, M. Li, and L. M. Ni, "Recognizing exponential inter-contact time in VANETs," in Proc. 29th IEEE INFOCOM, San Diego, CA, Mar. 14-19, 2010, pp. 1-5.

[25] E. Altman, G. Neglia, F. De Pellegrini, and D. Miorandi, "Decentralized stochastic control of delay tolerant networks," in Proc. 28th IEEE INFOCOM, Rio de Janeiro, Brazil, Apr. 19-25, 2009, pp. 1134-1142.

[26] S. Ioannidis, A. Chaintreau, and L. Massoulié, "Optimal and scalable distribution of content updates over a mobile social network," in Proc. 28th IEEE INFOCOM, Rio de Janeiro, Brazil, Apr. 19-25, 2009, pp. $1422-1430$.

[27] A. Chaintreau, J. Y. Le Boudec, and N. Ristanovic, "The age of gossip: Spatial mean field regime," in Proc. ACM SIGMETRICS, Seattle, WA, Jun. 15-19, 2009, pp. 109-120.

[28] M. H. R. Khouzani, S. Sarkar, and E. Altman, "Maximum damage malware attack in mobile wireless networks," in Proc. 29th IEEE INFOCOM, San Diego, CA, Mar. 15-19, 2010, pp. 1-9.

[29] L. Hu, J. Y. Le Boudec, and M. Vojnovi, "Optimal channel choice for collaborative ad-hoc dissemination," in Proc. 29th IEEE INFOCOM, San Diego, CA, Mar. 15-19, 2010, pp. 109-120.

[30] R. R. Darling and J. R. Norris, "Differential equation approximations for Markov chains," Probab. Surveys, vol. 5, pp. 37-79, 2008.

[31] K. Kar, A. Krishnamurthy, and N. Jaggi, "Dynamic node activation in networks of rechargeable sensors," in Proc. 24th IEEE INFOCOM, Miami, FL, Mar. 13-17, 2005, pp. 1997-2007.

[32] C. M. Fonseca and P. J. Fleming, "An overview of evolutionary algorithms in multiobjective optimization," Evol. Comput., vol. 3, no. 1, pp. 1-16, 1995.

[33] A. Keränen, J. Ott, and T. Kärkkäinen, "The ONE simulator for DTN protocol evaluation," in Proc. 2th Int. Conf. Simulation Tools Tech., Rome, Italy, Mar. 2-6, 2009, pp. 1-10.

[34] B. S. Everitt, The Cambridge Dictionary of Statistics, 3rd ed. Cambridge, U.K.: Cambridge Univ. Press, 2006.

[35] Shanghai Taxi Trace Data. [Online]. Available: http://wirelesslab.sjtu. edu.cn/

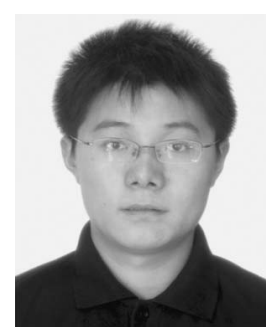

Yong Li (M'09) received the B.S. degree from Huazhong University of Science and Technology, Wuhan, China, in 2007. He is currently working toward the Ph.D. degree with the State Key Laboratory on Microwave and Digital Communications, Tsinghua National Laboratory for Information Science and Technology, Department of Electronic Engineering, Tsinghua University, Beijing, China.

His research interests include mobile delaytolerant networks; forwarding policies design, buffer management design and performance evaluation; mobility modeling; mobility management in next-generation wireless Internet Protocol networks; Mobile internet protocols (IP), session initiation protocol, Proxy mobile IP, cross-layer design, multicast mobility, modeling for mobility performance evaluation, and enhancing handoff performance enhancement; and mobility management architecture proposals.

$\mathrm{Mr}$. Li serves as a paper reviewer for the IEEE International Conference on Communications and the, Vehicular Technology Conference; the International Conference on Information Networking; the Personal, Indoor, and Mobile Radio Communications Conference; the Asia-Pacific Conference on Communications; etc. 


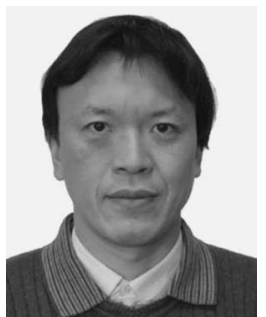

Zhaocheng Wang (M'09-SM'11) received the B.S., M.S., and Ph.D. degrees from Tsinghua University, Beijing, China, in 1991, 1993, and 1996, respectively.

From 1996 to 1997, he was with Nanyang Technological University, Singapore, as a Postdoctoral Fellow. From 1997 to 1999, he was with OKI Techno Centre Pte. Ltd., Singapore, first as a Research Engineer and then as a Senior Engineer. From 1999 to 2009, he was with SONY Deutschland GmbH, first as a Senior Engineer and then as a Principal Engineer. He is currently a Professor with the State Key Laboratory on Microwave and Digital Communications, Tsinghua National Laboratory for Information Science and Technology, Department of Electronic Engineering, Tsinghua University. He is the author or coauthor of more than 60 technical papers. He is the holder of 22 granted U.S./European-Union patents. His research interests include wireless communications, digital broadcasting, and millimeter-wave communications.

Dr. Wang has served as a technical program committee co-chair/member of many international conferences. He is a Fellow of the Institution for Engineering and Techonology.

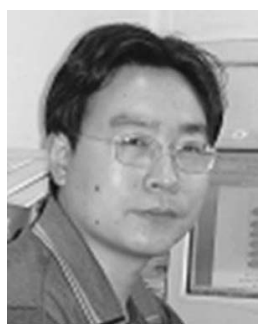

Depeng Jin (M'09) received the B.S. and Ph.D. degrees in electronics engineering from Tsinghua University, Beijing, China, in 1995 and 1999, respectively.

He is currently an Associate Professor with State Key Laboratory on Microwave and Digital Communications, Tsinghua National Laboratory for Information Science and Technology, Department of Electronic Engineering, Tsinghua University, and the Vice Chair of the Department of Electronic Engineering. His research interests include telecommunications, high-speed networks, application-specific integrated circuit design, and future Internet architectures.

Dr. Jin was the recipient of the National Scientific and Technological Innovation Prize (Second Class) in 2002.

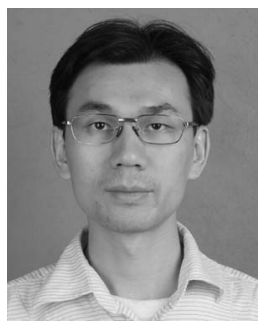

Li Su received the B.S. degree in electronics engineering from Nankai University, Tianjin, China, in 1999 and the Ph.D. degree in electronics engineering from Tsinghua University, Beijing, China, in 2007.

$\mathrm{He}$ is currently a Research Associate with the State Key Laboratory on Microwave and Digital Communications, Tsinghua National Laboratory for Information Science and Technology, Department of Electronic Engineering, Tsinghua University. His research interests include telecommunications, future Internet architectures, and on-chip networks.

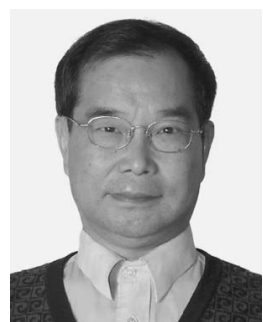

Lieguang Zeng received the B.S. degree from Tsinghua University, Beijing, China, in 1970.

Since 1970, he has been with the State Key Laboratory on Microwave and Digital Communications, Tsinghua National Laboratory for Information Science and Technology, Department of Electronic Engineering, Tsinghua University, where he is currently a Professor. He has published more than 150 research papers. He is the holder of four patents. His research interests include telecommunications, high-speed networks, application-specific integrated circuit design, and future Internet architectures.

Prof. Zeng received the National Scientific and Technological Innovation Prize (Second Class) in 1987, 1991, and 2002, as well as provincial- and ministerial-level science and technology progress prizes eight times.

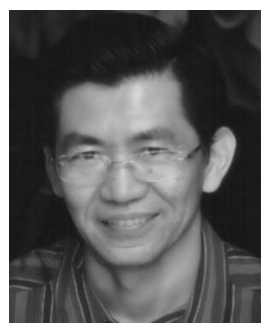

Sheng Chen (M'90-SM'97-F'08) received the B.Eng. degree in control engineering from East China Petroleum Institute, Dongying, China, in 1982, the Ph.D. degree in control engineering from the City University, London, U.K., in 1986, and the D.Sc. degree from the University of Southampton, Southampton, U.K., in 2005.

From 1986 to 1999, he held research and academic appointments with the Universities of Sheffield, Edinburgh, and Portsmouth, all in the U.K. Since 1999, he has been with Department of Electronics and Computer Science, Faculty of Physical and Applied Sciences, University of Southampton, where he currently holds the post of Professor of intelligent systems and signal processing. He is a Distinguished Adjunct Professor with the Faculty of Engineering, King Abdulaziz University, Jeddah, Saudi Arabia. He has published more than 450 research papers. His recent research interests include adaptive signal processing, wireless communications, modeling and identification of nonlinear systems, neural network and machine learning, intelligent control system design, evolutionary computation methods, and optimization

Dr. Chen is a Fellow of the Institution of Engineering and Technology and a Chartered Engineer. In the database of the world's most highly cited researchers in various disciplines, compiled by the Institute for Scientific Information, he is on the list of the highly cited researchers in the engineering category (March 2004). 